\title{
An exact formalism to study the thermodynamic properties of hard-sphere systems under spherical confinement
}

\author{
Ignacio Urrutia \\ Consejo Nacional de Investigaciones Científicas y Técnicas, \\ Argentina (CONICET) and Departamento de Fúsica, Comisión Nacional de Energía Atómica, \\ Av. Gral. Paz 1499 (RA-1650) San Martín, Buenos Aires, Argentina.* \\ Gabriela Castelletti \\ Instituto de Astronomía y Física del Espacio (CONICET-UBA), \\ Ciudad universitaria, Cdad. Aut. de Buenos Aires, Argentina.
}

\begin{abstract}
This paper presents a modified grand canonical ensemble which provides a new simple and efficient scheme to study few-body fluid-like inhomogeneous systems under confinement. The new formalism is implemented to investigate the exact thermodynamic properties of a hard sphere (HS) fluid-like system with up to three particles confined in a spherical cavity. In addition, the partition function of this system was used to analyze the surface thermodynamic properties of the many-HS system and to derive the exact curvature dependence of both the surface tension and adsorption in powers of the density. The expressions for the surface tension and the adsorption were also obtained for the many-HS system outside of a fixed hard spherical object. We used these results to derive the dependence of the fluid-substrate Tolman length up to first order in density.
\end{abstract}

\footnotetext{
*iurrutia@cnea.gov.ar
} 


\section{INTRODUCTION}

The thermodynamic and statistical mechanical properties of a fluid composed of many particles confined in small pores have been extensively investigated from both a theoretical and experimental point of view [1-3]. In these systems the fluid fills the cavity inhomogeneously and under extreme conditions, the confinement is responsible for the dimensional crossover of the particles [4]. On the contrary, the knowledge about the statistical mechanical properties of fluid-like systems composed of few bodies is rather poor. Notoriously, few-body systems have played a key role in the development of various fields of physical science including classical, relativistic and quantum mechanics. Of course, the fundamental advantage of the few-body systems over the many-body ones is that only the former are (in most cases) analytically tractable. In this sense we claim that, the importance of studying few-body systems within the framework of the statistical mechanics of inhomogeneous fluids relies in the fact that it provides exact solutions in a subject where exact results are quite scarce. We find this fact remarkable because these solutions provide new insights to better understand the properties of many-body systems.

The grand canonical ensemble (GCE), in which the volume, temperature and chemical potential are fixed, is certainly the most used scheme in the formulation of theories of fluids. In particular, the density functional theory (DFT) formulated in the GCE, is one of the most successful approaches used to describe the properties of inhomogeneous fluids. Indeed, DFT calculations make it possible to analyze the adsorption of fluids on substrates and in porous matrices as well as study the interfacial phenomena. Other theories as the scaled particle theory (SPT) are also formulated in the GCE. In this context hard spheres (HS) systems play a distinguished role in developing of both, DFT and SPT approaches, and perturbation theories of fluids [5]. Moreover, HS systems are of particular interest as they constitute a simplified model for both simple fluids and colloidal particles [6].

In this work we present a novel formulation that makes it possible the study of few-body inhomogeneous fluids using a GCE scheme in which the maximum number of particles is considered as a parameter of the system. We employ the proposed GCE to perform an exact study of the HS system with at most three particles confined in a spherical cavity. In addition, on the basis of the new results, we analyze the surface thermodynamic properties of the many-HS system spherically confined. We present our result for the exact curvature dependence of surface (or boundary) tension and adsorption, obtained in powers of the density. The formulation proposed here is found to be a valuable tool for describing highly confined fluids constituted by few bodies. Moreover, it will potentially serve to improve the DFT and SPT descriptions associated with the surface-related thermodynamic properties and their curvature dependence.

\section{PARTITION FUNCTION OF THE FEW-BODY OPEN SYSTEM}

Consider a one-component fluid containing a non-fixed number of particles which evolve inside a region of the space that we will refer to as $\mathcal{B}$. Such a region is determined by an external potential $\varphi(\mathbf{r})$ that takes finite values in $\mathcal{B}$ and diverges outside it. The boundary of $\mathcal{B}$ is assumed to be at a constant and uniform temperature $T$. In these circumstances we expect the fluid will attain an equilibrium state in which its physical properties either remain constant or fluctuate around a fixed value. Furthermore, for an inhomogeneous fluid system in an equilibrium state we make the following usual assumptions: i) if the exact expression for the grand canonical partition function (GCPF) $\Xi$ is known, then the exact grand potential function is given by

$$
\Omega=-\beta^{-1} \ln \Xi
$$

where $\beta=\left(k_{B} T\right)^{-1}$ is the inverse temperature (being $k_{B}$ and $T$ the Boltzmann's constant and the temperature, respectively); ii) a given thermodynamic property of the system, namely $X$, can be identified with its mean (Gibbsian) ensemble value $\langle X\rangle$. Additionally, it is expected that $\langle X\rangle$ to be equal to the time averaged value of $X$ over an interval $\tau, \bar{X}_{\tau}=\frac{1}{\tau} \int_{\tau} X(t) d t$ (at least for a value of $\tau$ large enough). Typically, $X$ may be the energy $E$, the pressure $p$, the number of particles $N$, etc. Standard statistical mechanical demonstrations show that the derivatives of the grand potential are related to the mean ensemble values $\langle X\rangle$ of most thermodynamic quantities. Other thermodynamic quantities may be computed from the grand potential and its derivatives by applying usual thermodynamic relations $[5,7]$.

In order to study few-body inhomogeneous systems, we now consider a container $\mathcal{B}$ with at most $\mathcal{M}$ particles, which in what follows will be a fixed parameter. The GCPF of this system is given by

$$
\Xi_{\mathcal{M}}=\sum_{j=0}^{\mathcal{M}} \mathrm{I}_{j} z^{j} Z_{j}
$$


where $\mathcal{M}$ is the cutoff value for the maximum number of particles. The indistinguishability factor $I_{j}$ is either equal to $\mathrm{I}_{j}=1 / j$ ! for indistinguishable particles or $\mathrm{I}_{j}=1$ for distinguishable particles. In addition, $z=\Lambda^{-3} \exp \beta \mu$ is the activity being $\Lambda=h /\left(2 \pi m k_{B} T\right)^{1 / 2}$ the thermal de Broglie wavelength, while $\mu, m$ and $h$ are the chemical potential, the mass of each particle and the Planck's constant, respectively. Finally, the configuration integral (CI) of a system with $j$ particles is

$$
Z_{j}=\int \ldots \int \prod e_{l} \prod e_{l m} d^{j} \mathbf{r}
$$

with $Z_{0}=1$. Here, $e_{l}=\exp \left[-\beta \varphi\left(\mathbf{r}_{l}\right)\right]$ and $e_{l m}=\exp \left[-\beta \phi\left(\mathbf{r}_{l m}\right)\right]$ are the Boltzmann's factors corresponding to the external and pair interactions, respectively. Note that, the integration domain in Eq. (3) is the complete space due to the fact that the spatial confinement of the particles in the region $\mathcal{B}$ is considered in the $e_{l}$ terms.

Because of $\Xi_{\mathcal{M}}$ is the GCPF of our system, in Eq. (1) we can replace $\Xi$ with $\Xi_{\mathcal{M}}$ to define $\Omega$. The knowledge of $Z_{j}$ for $1 \leq j \leq \mathcal{M}$ along with Eq. (1) and Eq. (2) provide the exact statistical-thermodynamic properties of the system. In Eq. (2), the expression for $\Xi_{\mathcal{M}}$ is an incomplete (or restricted) version of the usual GCPF obtained from $\Xi=\lim _{\mathcal{M} \rightarrow \infty} \Xi_{\mathcal{M}}$. A different restricted GCE was introduced by Yang and Lee [8] in their study of the condensation of gases. It was used later by Woods et al. [9] to study the adsorption of fluids in cavities. We emphasize that in their analysis $\mathcal{M}$ represents the maximum number of particles that fit in $\mathcal{B}$, instead of an externally imposed parameter as we have assumed in our formalism. An interesting property of $\Xi_{\mathcal{M}}$, Eq. (2), is that for a cavity $\mathcal{B}$ with a fixed size and temperature we obtain $\lim _{z \rightarrow \infty} \Xi_{\mathcal{M}} \propto I_{j} z^{j} Z_{j}$ where $j$ denotes the maximum number of particles that hold in $\mathcal{B}$ (under the constraint $1 \leq j \leq \mathcal{M}$ ).

\section{THE FEW HS SYSTEM IN A SPHERICAL CONFINEMENT}

Few-body fluid-like systems of HS with one or two particles confined in cavities with different geometries, including the spherical, cuboidal and cylindrical cases, were analytically solved in the canonical ensemble in Refs. [10-12]. Those works make it possible to explore on exact grounds the corresponding open systems with $\mathcal{M}=2$. Further, the threebody HS system was exactly solved for the case of a spherical confinement [13, 14]. In the current work we focus on the study of the HS open system with $\mathcal{M}=3$ in a spherical cavity. Inhomogeneous systems with a spherical geometry are ubiquitously present in nature in the form of bubbles and drops. One remarkable characteristic of this symmetry is that it does not place any preferential direction on the space. In spite of the relevance of the spherical geometry several fundamental questions are still unresolved regarding the curvature dependence of the thermodynamic properties in spherical systems. In view of this, much efforts have been directed in recent years to address this fundamental issue. In our description we consider an external hard-wall potential, which is null in a spherical region $\mathcal{B}$ and diverges outside it. The Boltzmann's factor is $e_{l}=\Theta\left(R-r_{l}\right)$, where $\Theta(r)$ is the Heaviside function, $r_{l}$ is the distance from the center of the pore to the particle $l$, and $R$ is the effective radius of the pore [13]. The volume of $\mathcal{B}$ is $V=Z_{1}=\frac{4 \pi}{3} R^{3}$ while $A=4 \pi R^{2}$ is the surface area of its boundary. By using the expressions for $Z_{2}$ and $Z_{3}$ presented in Refs. $[10,13,14]$ and assuming indistinguishable particles, i.e., $I_{j}=1 / j$ !, we obtain the exact expression for $\Xi_{3}$

$$
\Xi_{3}=1+Z_{1} z+Z_{2} \frac{z^{2}}{2}+Z_{3} \frac{z^{3}}{6} .
$$

After fixing the diameter of the particles to be $\sigma=1$ to lighten our notation, the expressions for $Z_{2}$ and $Z_{3}$ are given by

$$
\begin{gathered}
Z_{2}= \begin{cases}\left(\frac{2 \pi}{3}\right)^{2}\left(R-\frac{1}{2}\right)^{3}\left(1+6 R+6 R^{2}+4 R^{3}\right), & \text { if } R \geq \frac{1}{2} \\
0, & \text { if } 0 \leq R<\frac{1}{2}\end{cases} \\
Z_{3}= \begin{cases}h-\Delta \tau, & \text { if } R \geq 1 \\
h, & \text { if } \frac{1}{\sqrt{3}} \leq R<1 \\
0, & \text { if } 0 \leq R<\frac{1}{\sqrt{3}},\end{cases}
\end{gathered}
$$

with

$$
\begin{aligned}
h= & \frac{\pi^{2}}{70}\left[\frac{1}{9} q\left(65+183 R^{2}-342 R^{4}+240 R^{6}\right)-\frac{9}{2} p_{1}\left(5+12 R^{2}\right)\right. \\
& \left.+p_{2} R\left(105-280 R^{2}+840 R^{4}-1152 R^{6}+640 R^{8}\right)\right]
\end{aligned}
$$




\begin{tabular}{|c|c|c|c|c|c|c|c|}
\hline$z$ & 0.1 & 0.2 & 0.5 & 1 & 2 & 5 & 10 \\
\hline$\rho_{b}$ & 0.072226 & 0.11583 & 0.18962 & 0.25142 & 0.31323 & 0.39028 & 0.44353 \\
\hline$z$ & 20 & 50 & 100 & 200 & 500 & $10^{3}$ & $10^{5}$ \\
\hline$\rho_{b}$ & 0.49217 & 0.54981 & 0.58888 & 0.62452 & 0.66704 & 0.69619 & 0.84313 \\
\hline
\end{tabular}

Table I. Activity values $z$, used for the curves plotted in Fig. 1 and the corresponding bulk density values, $\rho_{b}$.

$$
\begin{gathered}
q=\sqrt{3 R^{2}-1}, \quad p_{1}=\arctan (2 q), \quad p_{2}=\arctan (q / R) \\
\Delta \tau=\frac{128}{189} \pi^{3}(R-1)^{5}\left(1+5 R+\frac{69}{10} R^{2}+5 R^{3}+R^{4}\right)
\end{gathered}
$$

Concerning the basic geometrical properties of the spherical confinement we find that, a cavity with $R<\frac{1}{2}$ can contain at most one particle, while for the cases $R<\frac{1}{\sqrt{3}} \doteq 0.57735, R<\sqrt{\frac{3}{8}} \doteq 0.612372$ and $R<\frac{1}{\sqrt{2}} \doteq 0.707107$, a maximum of two, three and four particles can be fitted in the cavity, respectively. These values imply that by evaluating $\Xi_{3}$ we have not only solved the restricted system with $\mathcal{M}=3$ and any value of $R$, but also we have obtained the exact solution of the unrestricted system for $R<\sqrt{\frac{3}{8}}$ because in this case $\Xi(R)=\Xi_{3}(R)$.

The thermodynamic fundamental relations for the grand potential are

$$
\begin{gathered}
\Omega=U-T S-\mu N, \\
d \Omega=-S d T-P_{W} A d R-N d \mu,
\end{gathered}
$$

here $S$ is the entropy, $d V$ is equal to $A d R, P_{W}$ represents the (mean) pressure on the wall also called the workpressure, and $d W=P_{W} d V$ is the total reversible work performed by the system on its environment. Given that the system is athermal, it is preferable to use temperature-independent quantities like $\beta \Omega$ to conduct the analysis of its properties. Therefore, some of the usual thermodynamic and statistical mechanical relations for systems under spherical confinement can be written as

$$
\begin{gathered}
\beta P_{W}=-\left.A^{-1} \frac{\partial \beta \Omega}{\partial R}\right|_{\beta, z}, \\
N \equiv\langle N\rangle=-\left.z \frac{\partial \beta \Omega}{\partial z}\right|_{\beta, R}, \\
\sigma_{N}^{2} \equiv\left\langle N^{2}\right\rangle-N^{2}=\left.z \frac{\partial N}{\partial z}\right|_{\beta, R}
\end{gathered}
$$

Here $\sigma_{N}$ is the standard deviation in the number of particles which quantifies the spontaneous fluctuation of $N$. Since the energy of the system is equal to that of the classical ideal gas, i.e., $\beta U=\frac{3}{2} N$, using Eqs. (10) and (13) we can also calculate $S$. At this stage, the exact properties obtained for the system are functions of $z$ and $R$. One important point is the fact that the few-body HS open system is in chemical equilibrium with a bulk HS fluid. Unfortunately, the exact properties of the bulk HS fluid are not analytically known, and thus, to present our results in terms of the bulk density $\rho_{b}$ (instead of $z$ ) we adopt the simple and accurate Carnahan-Starling's equation of state, which for $z$ gives $[4,15]$

$$
z_{\mathrm{CS}}=\rho_{b} \times \exp \left[\frac{8 \eta_{b}-9 \eta_{b}^{2}+3 \eta_{b}^{3}}{\left(1-\eta_{b}\right)^{3}}\right]
$$

where $\eta_{b}=\frac{\pi}{6} \rho_{b} \sigma^{3}$ is the corresponding packing fraction. We remark that Eq. (15) is the unique approximated relation introduced in this section. Although it is exact only up to third order in density accurately describes the bulk system for all the fluid-phase density range. As a reference, we mention that in the bulk HS system the fluid (disordered) 


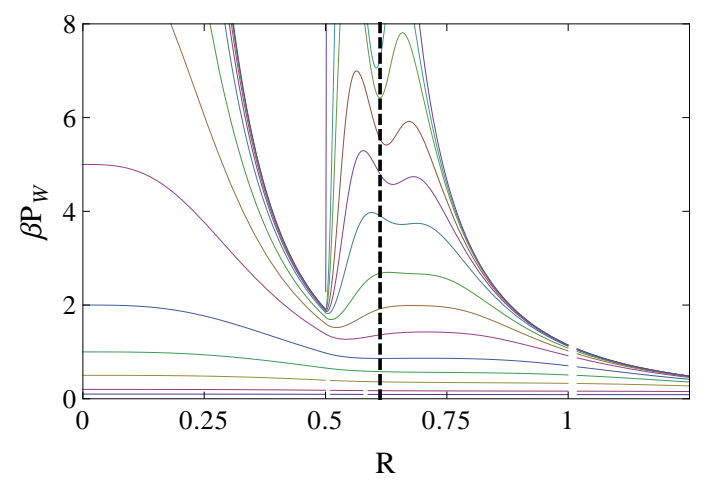

Figure 1. Pressure on the wall against the radius of the pore obtained for values of the activity $z$, and bulk density $\rho_{b}$ listed in Table I ( $z$ and $\rho_{b}$ increase from bottom to top). The dashed line indicates the radius $R=0.612$ for which a maximum of three particles can be fitted in the cavity.

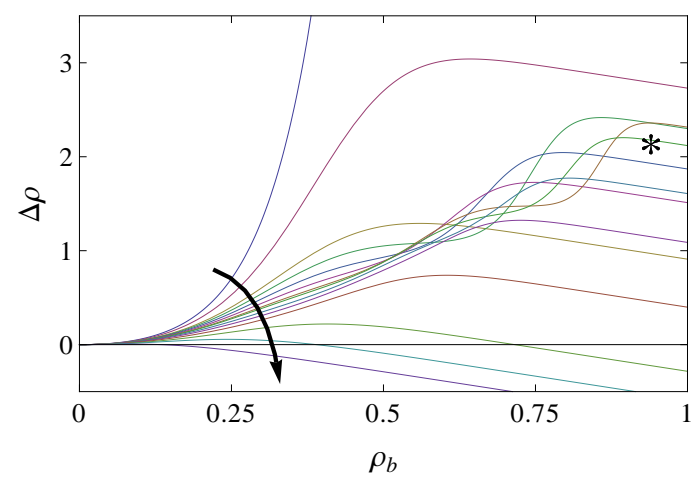

Figure 2. Relative adsorption in the pore as a function of the bulk density plotted for different radii of the pore $R=0.2,0.4,0.5$, $0.525,0.55,0.575,0.6,0.61237,0.65,0.7,0.8,1,1.2$, and 1.5 . The arrow marks the direction of increasing values of $R$. The asterisk indicates the curve for $R=0.61237$.

and solid (ordered) stable phases coexist at $\beta P \simeq 11.576$, being the fluid and the solid bulk-densities $\rho_{b f} \simeq 0.943$ and $\rho_{b s} \simeq 1.041$, respectively [16, 17].

Fig. 1 displays the pressure on the wall as a function of the radius of the cavity, considering different values of $\rho_{b}$. Table I summarizes the exact values of $z$ used in plotting each curve together with the corresponding approximated values of $\rho_{b}$ obtained from the numerical inversion of Eq. (15). The dashed vertical line in Fig. 1 corresponds to $R=0.612$, that is, the maximum value for which our results are exact even for the unrestricted system. From this graphic it is evident that the pressure on the wall has a non-monotonic behavior with loops characterized by regions of negative and positive slopes, which are related to the mechanical instability of the system. In Fig. 2 we plot the relative adsorption in the pore per unit volume as a function of $\rho_{b}$, that is $\Delta \rho=\hat{\rho}-\rho_{b}$ where $\hat{\rho}=N / V$. There, the arrow indicates increasing values of $R$. The negative adsorption in the case of the curves corresponding to $R=1,1.2$, and 1.5 is a consequence of the cutoff in the maximum number of particles contained in the pore. This also applies to regions with positive adsorption and negative slope observed in this plot for $R=0.65,0.7,0.8$, and 1 . Fig. 3 shows the filling of the cavity as a function of $\rho_{b}$ for different radii of the pore. For each value of $R$ considered the saturation value of $N$ corresponds to the maximum number of particles that holds in the cavity (at most three particles). As it can be observed, $N\left(\rho_{b}, R\right)$ is a non-decreasing function. It is noticeable that for $0.5<R<0.61237$ the curves change their concavity several times with the increase of $\rho_{b}$. This property determines the behavior of $\sigma_{N}^{2}\left(\rho_{b}\right)$ through Eq. (14). Fig. 4 illustrates the relation between the mean number of particles in the cavity and its fluctuation $\sigma_{N}$. Each curve corresponds to the parametric plot $\left(N(z), \sigma_{N}(z)\right)$ for a fixed value of $R$. We find that all the curves corresponding to $R<0.5$ collapse onto the semi-circumference centered at $N=0.5$. For $0.5<R<0.577$ and $\mathrm{N}$ increasing in value $1.5<N \leq 2$ the curves approach to the second semi-circumference centered at $N=1.5$. In the range $2.5<N \leq 3$ a third semi-circumference centered at $N=2.5$ is fitted by the curves corresponding to $R>0.577$. In addition, we have analyzed the case $\mathcal{M}=2$ and obtained a picture similar to that displayed for the case $\mathcal{M}=3$ but with only two semi-circumferences centered at $N=0.5$ and $N=1.5$ (the graphic is not shown here). One important 


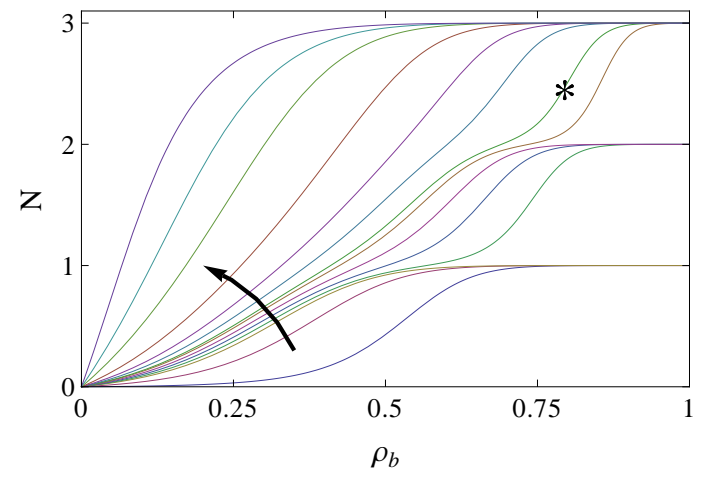

Figure 3. Mean number of particles vs. bulk density for different radii of the pore. The arrow, the asterisk and the values of $R$ were described in Fig. 2 .

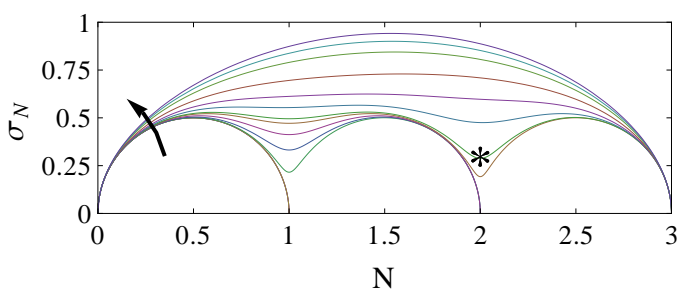

Figure 4. Standard deviation in $N$ as a function of $N$ for different radii of the pore. The arrow, the asterisk and the values of $R$ were described in Fig. 2 .

consequence of the graphic shown in Fig. 4 is that it can be straightforwardly extended to obtain the representation for the case with a value $\mathcal{M}>3$. Basically, it will involve a sequence of $\mathcal{M}$ semi-circumferences of unit diameter centered at half-integer values of $N$ along the abscissa axis. In particular, for a given $R$ the curve will start at the origin $\left(N, \sigma_{N}\right)=(0,0)$ and will collapse either onto the semi-circumference that ends at the value $N$ equal to the maximum number of particles that fit in the cavity or onto the $\mathcal{M}$-th semi-circumference in case $R$ is large enough.

It is to be noted that the outmost curve which connects $N=0$ with $N=\mathcal{M}$ can be analytically obtained by considering the limit of a very large cavity, $V \rightarrow \infty$ (infinite dilution). In this low density limit we can replace $Z_{i}$ with $Z_{1}^{i}=V^{i}=\left(\frac{4 \pi}{3} R^{3}\right)^{i}$ and hence $N$ and $\sigma_{N}$ become functions only of the parameter $a=z Z_{1}$. By following this procedure we effectively reduce the system to an ideal gas (with up to $\mathcal{M}$ particles). The exact analytic expressions for $\sigma_{N}(a)$ and $N(a)$ with $\mathcal{M}=3$ are parametrically plotted (using $a$ as a parameter) in the outmost curve in Figure 4. As a closing remark, this analysis shows that any system with at most one particle is described by the first semi-circumference, irrespective of the nature of the particle and the confinement.

\section{THE MANY HS SYSTEM ONTO A SPHERICAL WALL}

In what follows we draw attention to the implications that the expression for $\Omega$, obtained for the system with $\mathcal{M}=3$, has on the surface-thermodynamic properties of the many-body system. In particular, we will address on the HS system in contact with a hard spherical wall in the low density limit. For each magnitude of the system, our main goal is to find its exact power series in $\rho_{b}$ up to the three-body term, with the dependence on the curvature included through the coefficients of the series. Our procedure is based on the ideas developed in Ref. [12], which enable us to express the analytically known terms $Z_{i}(R)$ as a function of the set of measures $\{V, A, R\}$. We begin by placing the dividing surface at $R$ and we use the same decomposition rule adopted in Ref. [13] (Eqs. (28) and (29) therein). For the unknown $Z_{i}$ terms we assume a generic dependence $Z_{i}(V, A, R)$. As a result, we obtain an expression for $\beta \Omega$ that is a function of $V, A, R$ and $z$. We then fix $\mathcal{M}=4$ in Eq. (2) and calculate the magnitude $X$. By following standard procedures based on the inversion and composition of the power series we expand the expression for $X$ as a power series in the variable $\rho_{b}$. Then, we truncate the series to the first term in which $Z_{4}$ appears. The exact series of $z\left(\rho_{b}\right)$ truncated to third order in density, $z=\rho_{b}+\frac{4 \pi}{3} \rho_{b}^{2}+\frac{47 \pi^{2}}{36} \rho_{b}^{3}$, is sufficient for our purposes as we have verified that the inclusion of the fourth order term does not modify our results. In addition, to obtain the planar-surface property we 


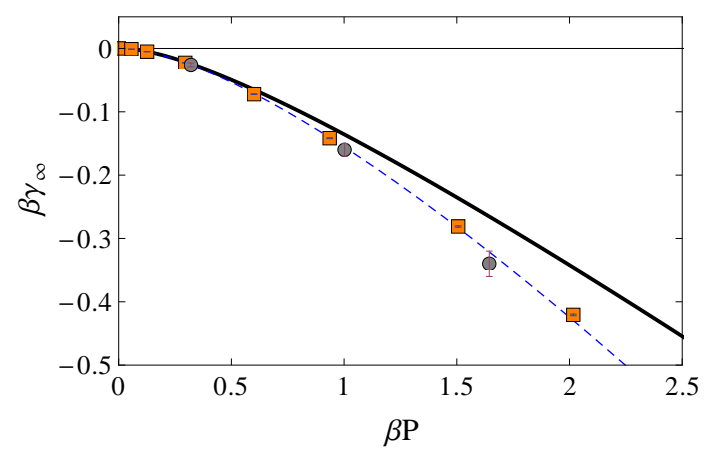

Figure 5. Fluid-substrate surface tension vs. pressure. The filled circles and squares correspond to simulation and thermodynamic integration data from [21] and [22], respectively. The dashed line shows SPT values [20]. The parametric plot of the power series $\beta P\left(\rho_{b}\right)$ and $\beta \gamma_{\infty}\left(\rho_{b}\right)$ is shown by the continuous line.

consider in the series the limit $R \rightarrow \infty$. Thus, we determine in the planar limit the expressions for the fluid-substrate surface tension $\gamma \equiv \partial \Omega /\left.\partial A\right|_{V, R}$ and the adsorption per unit area $\Gamma \equiv V\left(\hat{\rho}-\rho_{b}\right) / A$,

$$
\begin{gathered}
\beta \gamma_{\infty}=-\frac{\pi}{8} \rho_{b}^{2}\left(1+\frac{149 \pi}{210} \rho_{b}\right)+O\left(\rho_{b}^{4}\right), \\
\Gamma_{\infty}=\frac{\pi}{4} \rho_{b}^{2}\left(1-\frac{113 \pi}{420} \rho_{b}\right)+O\left(\rho_{b}^{4}\right) .
\end{gathered}
$$

Eqs. (16) and (17) exactly reproduce the first two terms (the only known up to present) of the series expansion of $\beta \gamma_{\infty}\left(\rho_{b}\right)$ and $\Gamma_{\infty}\left(\rho_{b}\right)[18,19]$ and are consistent with the Gibbs adsorption isotherm $\Gamma_{\infty}=-\partial \gamma_{\infty} / \partial \mu$ to order $\rho_{b}^{3}$.

In Fig. 5 we show a plot of the surface tension vs. pressure in the low pressure region, which is also the low-density region. Our results are represented parametrically $\left(\beta \gamma_{\infty}\left(\rho_{b}\right), \beta P\left(\rho_{b}\right)\right)$ using both, Eq. (16) and the exact third order virial series $\beta P=\rho_{b}+\frac{2 \pi}{3} \rho_{b}^{2}+\frac{5 \pi^{2}}{18} \rho_{b}^{3}$. The dashed line included in this figure plots previous SPT results taken from Fig. 2 in Ref. [20]. Since all the plotted SPT versions are nearly indistinguishable in the range of pressure considered, we only include the results of the CS-SPT and CS-SPT $M$ (they collapse on a unique curve) to make easy the examination of consistency between SPT and our result. Also, the simulation and thermodynamic integration data from [21, 22] are indicated by filled circles and squares. We note that simulation results fall nicely on the SPT curve. On the other hand, the exact low-density series adequately fits numerical results over the range $0<\beta P \lesssim 0.6$.

As it is well known, the relevant problem in statistical mechanics regarding the dependence of the surface thermodynamic properties on the curvature remains unresolved, even for the HS fluid in contact with hard spherical walls. However, following the procedure described above and writing the magnitude $X / X_{\infty}$ as a double power series in the variables $R^{-1}$ and $\rho_{b}$, we obtain

$$
\begin{aligned}
& \gamma(R) / \gamma_{\infty}=1+\delta_{\infty} 2 R^{-1}+\delta_{\mathrm{k}} R^{-2}+O\left(R^{-3}\right), \\
& \Gamma(R) / \Gamma_{\infty}=1+\xi_{\mathrm{j}} 2 R^{-1}+\xi_{\mathrm{k}} R^{-2}+O\left(R^{-3}\right) .
\end{aligned}
$$

The complete expressions for $\gamma(R)$ and $\Gamma(R)$ in the limit of both small $\rho_{b}$ and large $R$ is presented in Appendix A. In the latter two equations, $2 R^{-1}$ and $R^{-2}$ are the mean and Gaussian curvatures of the spherical cavity, respectively. The coefficient $\delta_{\infty}$ denotes the radius-independent wall-fluid Tolman length. In fact, neither of the coefficients in these equations depends on $R$. We verified that Eq. (18) and Eq. (19) hold for fluids in contact with a hard spherical wall, irrespective of whether the fluid is inside the cavity $(R>0)$ or outside of a fixed hard sphere $(R<0$, also known as an empty cavity in a bulk fluid) [13]. To first order in density the coefficients $\delta_{\infty}$ and $\delta_{k}$ can be computed from

$$
\begin{aligned}
\delta_{\infty} & \doteq 0.34299 \rho_{b}, \delta_{\mathrm{k}} \doteq-0.05555+0.19933 \rho_{b}, \\
\xi_{\mathrm{j}} & \doteq 0.51448 \rho_{b}, \xi_{\mathrm{k}} \doteq-0.05555+0.29899 \rho_{b} .
\end{aligned}
$$

No numerical simulation results have been published up to present for these magnitudes. The expressions for both $\delta_{\infty}$ and $\delta_{\mathrm{k}}$ determined by the above procedure are consistent with those recently reported in [13], derived from an 


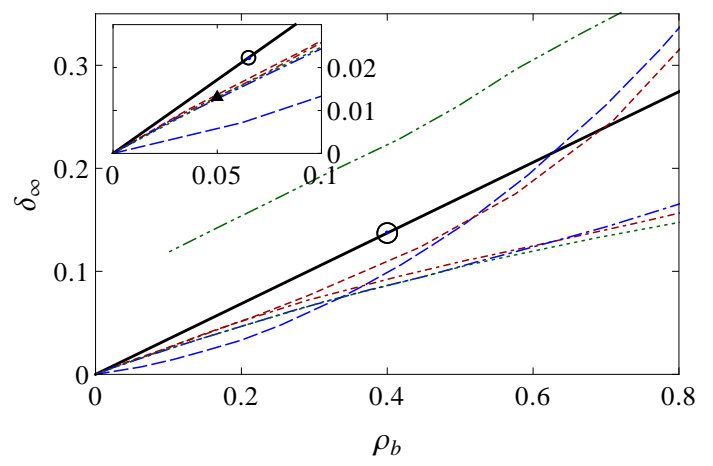

Figure 6. Tolman length vs. density. The continuous curve with the open circle corresponds to the linear dependence in $\rho_{b}$ from this work. The six SPT versions from Refs. [20,23] are shown by non-continuous curves: $\mathrm{SPT}_{3}$ (dotted line), $\mathrm{SPT}_{6}$ (short dashed line), CS-SPT (dashed line), $\mathrm{SPT}_{6 M}$ (short dashed-dot line), CS-SPT $M$ (dashed-dot line), and ESPT (dashed-dot-dot line that lies above all other lines). The graphic includes at the top left corner a close up of the curves around $\rho_{b}=0$.

exact calculation for a few-body system treated within the framework of the canonical ensemble. Figure 6 displays the Tolman length vs. density. There, the continuous line with the open circle shows the power series of $\delta_{\infty}\left(\rho_{b}\right)$ around $\rho_{b}=0$ truncated to the linear order, Eq. (20). This figure also includes the estimates of $\delta_{\infty}$ obtained by Siderius et al. [20] and Chatterjee et al. [23] using six different versions of SPT, which are plotted using different styles of non-continuous lines. The first observation we make is that the general trend of the SPT curves is well reproduced by our result. In what follows we will concentrate in the low density region of this figure for which we know the exact values of the $\delta_{\infty}$-intercept and the slope at $\rho_{b}=0$ of the true $\delta_{\infty}\left(\rho_{b}\right)$ function. By adopting the nomenclature used in Ref. [20], we note that the SPT proposals CS-SPT $M, \mathrm{SPT}_{6}, \mathrm{SPT}_{6 M}$ and $\mathrm{SPT}_{3}$ are nearly indistinguishable as $\rho_{b}$ becomes 0 . In view of this we address our analysis of these four curves directly to the study of CS-SPT $M$. Interestingly, among the six SPT theories, the CS-SPT and CS-SPT $M$ are the unique ones that provide analytic formulae for $\delta_{\infty}\left(\rho_{b}\right)$, and thus we can determine the corresponding series expansion. For CS-SPT and CS-SPT $M$ we obtain slopes of 0.0970 and 0.2675 , respectively. The comparison of CS-SPT and its modified version CS-SPT $M$ with the exact result, 0.34299 , reveals that the modified approach to SPT significantly improves the description of the $\delta_{\infty}$ behavior at low density. Regarding the ESPT curve for $\delta_{\infty}\left(\rho_{b}\right)$, its behavior is unknown in the range $0<\rho_{b}<0.1$.

\section{SUMMARY}

In this work we have introduced a new formalism to analyze the statistical mechanics of few-body open systems. It is based on a grand canonical ensemble with a restriction in the maximum number of particles. The main feature of the new GCE formalism is that it incorporates the fluctuation in the number of particles, measured by $\sigma_{N}$. On the basis of this new grand canonical scheme we have calculated the partition function of the HS fluid-like system composed of at most three particles confined in a spherical cavity. Using this partition function we obtained the analytic expressions for several thermodynamic properties of the system. Among all the properties analyzed for this system, the dependence of $\sigma_{N}$ on $N$ is, in virtue of its simplicity, symmetry and beauty, the most interesting one. We are convinced that it should deserve more attention in future studies about the statistical mechanics of confined fluids.

We have also investigated the thermodynamic properties of the many-body system of HS in contact with a hard spherical wall. By adopting a power series representation we reported new expressions for both the surface tension and adsorption, as a function of the density and curvature. In the planar wall limit $R \rightarrow \infty$ our results are consistent with those from previous results published in the literature. We used the new expression for the surface tension to obtain the fluid-wall Tolman length up to the first order in density. We conclude that the general trend of $\delta_{\infty}$ derived from the new formalism adequately agrees with SPT results presented by Siderius et. al. [20]. In particular, from the comparison of our exact result with that obtained within the framework of SPT, we found that the highest level of consistency in $\delta_{\infty}\left(\rho_{b}\right)$ at zero density occurs for the CS-SPT $M, \mathrm{SPT}_{6}, \mathrm{SPT}_{6 M}$, and $\mathrm{SPT}_{3}$ versions although the slope obtained in these cases differs by about $20 \%$ from the exact value. We feel that our findings concerning the description of HS systems are important as they represent new analytic results that contribute to better understand the longstanding problem of the thermodynamics of confined HS systems and their surface-related properties. Moreover, our results may provide new insights for future improvements on DFT which are based on a reference fluid of HS. 


\section{ACKNOWLEDGMENTS}

I.U. and G.C. acknowledge the comments from the anonymous referees. This work was supported by Argentina Grants CONICET-PIP 0546, UBACyT 20020100200156 and ANPCyT-PICT 2008-0795.

\section{Appendix A:}

We present here the expressions for $\beta \gamma$ and $\Gamma$ up to the third order in density

$$
\begin{gathered}
\beta \gamma=\left(a_{2}-\frac{c_{2 k}}{R^{2}}\right) \rho_{b}{ }^{2}+\left(a_{3}+\frac{8}{3} \pi a_{2}-\frac{2 c_{3 j}}{R}-\frac{3 c_{3 k}+8 \pi c_{2 k}}{3 R^{2}}\right) \rho_{b}{ }^{3}, \\
\Gamma=\left(-a_{2}+\frac{c_{2 k}}{R^{2}}\right) 2 \rho_{b}{ }^{2}+\left(-\frac{9 a_{3}+16 \pi a_{2}}{3}+\frac{8 c_{3 j}}{R}+\frac{9 c_{3 k}+16 \pi c_{2 k}}{3 R^{2}}\right) \rho_{b}{ }^{3},
\end{gathered}
$$

with $a_{2}=-\frac{\pi}{8}, a_{3}=\frac{137 \pi^{2}}{560}, c_{2 k}=-\frac{\pi}{2^{4} 3^{2}}, c_{3 j}=\frac{9 \pi \sqrt{ } 3+16 \pi^{2}}{1536}, c_{3 k}=\frac{781 \pi^{2}}{36288}$. In both equations the coefficients of $\rho_{b}{ }^{3}$ were truncated at order $R^{-2}$.

[1] J. Dunkel and S. Hilbert, Physica A: Statistical Mechanics and its Applications 370, 390 (2006)

[2] A. V. Neimark and A. Vishnyakov, The Journal of Physical Chemistry B 110, 9403 (2006)

[3] E. Perret, K. Nygård, D. K. Satapathy, T. E. Balmer, O. Bunk, M. Heuberger, and J. F. v. d. Veen, Journal of Physics: Condensed Matter 22, 235102 (2010)

[4] A. Gonzalez, J. A. White, F. L. Román, and S. Velasco, The Journal of Chemical Physics 125, 064703 (2006)

[5] J. P. Hansen and I. R. McDonald, Theory of simple liquids, 3rd Edition (Academic Press, Amsterdam, 2006)

[6] R. Roth, Journal of Physics: Condensed Matter 22, 063102 (2010)

[7] T. L. Hill, Statistical Mechanics (Dover, New York, 1956)

[8] C. N. Yang and T. D. Lee, Phys. Rev. 87, 404 (1952)

[9] G. B. Woods, A. Z. Panagiotopoulos, and J. S. Rowlinson, Molecular Physics 63, 49 (1988)

[10] I. Urrutia, Journal of Statistical Physics 131, 597 (2008), arXiv:cond-mat/0609608

[11] I. Urrutia and L. Szybisz, Journal of Mathematical Physics 51, 033303 (2010), arXiv:0909.0246

[12] I. Urrutia, The Journal of Chemical Physics 133, 104503 (2010), arXiv:1005.0723

[13] I. Urrutia, The Journal of Chemical Physics 135, 024511 (2011)

[14] I. Urrutia, The Journal of Chemical Physics 135, 099903 (2011)

[15] N. F. Carnahan and K. E. Starling, The Journal of Chemical Physics 51, 635 (1969)

[16] J. Kolafa, S. Labík, and A. Malijevský, Phys. Chem. Chem. Phys. 6, 2335 (2004)

[17] M. N. Bannerman, L. Lue, and L. V. Woodcock, Journal of Chemical Physics 132, 084507 (2010)

[18] A. Bellemans, Physica 28, 493 (1962)

[19] D. W. Siderius and D. S. Corti, Industrial \& Engineering Chemistry Research 45, 5489 (2006), http://pubs.acs.org/doi/pdf/10.1021/ie051038t

[20] D. W. Siderius and D. S. Corti, Journal of Chemical Physics 127, 144502 (2007)

[21] M. Heni and H. Löwen, Journal of Physics: Condensed Matter 13, 4675 (2001)

[22] B. B. Laird and R. L. Davidchack, The Journal of Chemical Physics 132, 204101 (2010)

[23] S. Chatterjee, P. G. Debenedetti, and F. H. Stillinger, The Journal of Chemical Physics 125, 204505 (2006) 